\title{
Nanoscale Internal Fields in a Biased
}

\section{Graphene-Insulator-Semiconductor Structure: Supplemental Information}

Sylvie Rangan, ${ }^{*}, \dagger$ Malathi Kalyanikar, ${ }^{\ddagger}$ Junxi Duan, ${ }^{\dagger}$ Gang Liu, ${ }^{\text {" Robert Allen }}$

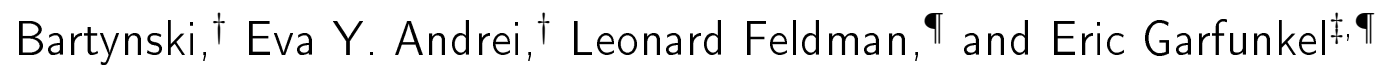

$\dagger$ Department of Physics and Astronomy, and Laboratory for Surface Modification, Rutgers University, 136 Frelinghuysen Road, Piscataway, New Jersey 08854, USA

$\$$ Department of Chemistry and Chemical Biology, Rutgers University, 610 Taylor Road, Piscataway, New Jersey 08854, USA

9Institute for Advanced Materials, Devices and Nanotechnology, Rutgers University, 607 Taylor Road, Piscataway, New Jersey 08854, USA

E-mail: rangan@physics.rutgers.edu

Phone: +1-848-445-8419 


\section{Experimental methods}

\section{Sample preparation}

The structure used in this study consisted of an $\mathrm{SiO}_{2}$-Si base structure onto which the top graphene contact layer was added. The semiconducting substrate was a commercially purchased degenerately doped (resistivity $<0.005 \mathrm{ohm} / \mathrm{cm}-10^{19}$ doping level) p-type $\operatorname{Si}(100)$ wafer which was cleaned by the RCA method to remove the thin native oxide layer. ${ }^{1} \mathrm{~A}$ thick, $300 \mathrm{~nm}$ oxide layer was then grown using conventional wet thermal oxidation. This thick oxide pad region facilitated observation (in an optical microscope) of the subsequently added graphene overlayer, as well as acting as an electrical buffer layer where electrodes could be placed without shorting the device. To create a window through the thick oxide where XPS measurement could be performed, a circular window approximately $400 \mu \mathrm{m}$ in diameter was lithographically patterned and the $\mathrm{SiO}_{2}$ in the patterned area was removed by wet etching. Subsequently, a fresh $6 \mathrm{~nm}$ thermal oxide layer was grown on the lithographically exposed Si in a dry oxygen atmosphere at $900^{\circ} \mathrm{C}$ for 3 minutes.

Graphene was CVD-grown on copper using a procedure previously reported. ${ }^{2,3}$ A thin layer of poly-methyl-methacrylate (PMMA) was spin-coated on the graphene-copper substrate and the substrate copper was etched away. The polymer layer was then used to transfer the graphene onto the patterned $\mathrm{SiO}_{2} / \mathrm{Si}$ substrate and the sample was annealed to enhance the graphene conformality on the oxide structures. After a chemical wash in acetone, most of the residual polymer was removed by annealing in a forming gas $\left(H_{2}\right.$-Ar) atmosphere at $350^{\circ} \mathrm{C}$. Complementary optical measurements and Raman spectroscopy were used to characterize the graphene on control samples. To make electrical contact to the graphene overlayer, a thin layer of $\mathrm{Ti}(5 \mathrm{~nm})$ followed by a thicker layer of $\mathrm{Au}(45 \mathrm{~nm})$ were e-beam evaporated onto the graphene over the thicker pad oxide region. These contacts were then connected electrically to an external contact for biasing and/or current measurements. 


\section{Electrical measurements}

To ensure that the patterned ultrathin oxide was of high quality and that the MOS structure was not shorted, current-voltage (I - V) studies were performed on control samples prepared in the same batch, and used to confirm leakage current and breakdown voltage of the thin oxide. The leakage current (for a maximum of $\pm 2.7 \mathrm{~V}$ ) observed across the dielectric when measured both in-vacuo (during XPS) and under atmosphere conditions was in the range of few nanoamperes (for an area of $1.25 \times 10^{-7} \mathrm{~m}^{2}$ ). The bias range was restricted during the XPS measurements on the real sample to $2.7 \mathrm{~V}$ to avoid possible breakdown of the dielectric. In our study, for a maximum potential (of $2.7 \mathrm{~V}$ ) across the dielectric, the field is $4.5 \mathrm{MV} / \mathrm{cm}$ (a high performance $\mathrm{SiO}_{2}$ based CMOS device can withstand up to $12 \mathrm{MV} / \mathrm{cm}^{4}$ ). The oxide preparation methodology determines how much field the dielectric can withstand before breakdown.

\section{Biased x-ray photoemission}

The XPS biasing experiments were preformed in a Thermo Scientific ESCA 250Xi system, equipped with a monochromated $\mathrm{Al} \mathrm{K} \alpha$ x-ray source $(\mathrm{h} \nu=1486.7 \mathrm{eV})$. XPS results were collected from an area of about $200 \mu \mathrm{m} \times 200 \mu \mathrm{m}$. In the studies reported here, the bottom of the Si substrate was grounded to the chamber and the bias was applied to the top graphene electrode via an external power supply. Multiple measurements across the biasing range were performed to ensure reproducibility of the results and to assess the error. Spectra were collected at several positions on the sample to confirm lateral bias homogeneity. Intensity variations of the x-ray source were taken into account by normalizing all photoemission peaks to the graphene $\mathrm{C}$ 1s signal. The gold electrode present in the sample was utilized as an internal reference for the binding energies $\left(\mathrm{Au} 4 \mathrm{f}_{7 / 2}\right.$ and $\mathrm{Au} 4 \mathrm{f}_{5 / 2}$ doublets were located at $84.0 \mathrm{eV}$ and $87.7 \mathrm{eV}$, respectively).

The attenuation lengths into $\mathrm{SiO}_{2}$ have been calculated using the NIST electron effective

attenuation-length database software, ${ }^{5}$ for an Al-K $\alpha$ photon source and a density of 2.3 
g. $\mathrm{cm}^{-3}$. The values obtained for electrons originating from the Si $2 \mathrm{p}$ and $\mathrm{O}$ 1s core levels, $35.3 \AA$ and $25.7 \AA$, respectively, are comparable to most recent studies. ${ }^{6}$ An oxide thickness of $60 \AA$ is calculated from the ratio of the $\mathrm{Si}^{4+}$ and $\mathrm{Si}^{0}$ photoemission intensities.

When measuring insulating films using photoemission, charging effects due to the creation of positive charges upon ionization need to be evaluated. In our case, as the oxide layer is connected to two conductive electrodes ( $\mathrm{Si}$ and $\mathrm{Gr}$ ), the effective length for charge extraction from the oxide layer is only $3 \mathrm{~nm}$, thus greatly reducing charging. ${ }^{7}$ Charging also depends on photon flux and might be time-dependent until a dynamic equilibrium is reached. In our case, no x-ray flux- or time- dependence was measurable, attributed to minimal charging for our structure.

\section{Additional XPS spectra}

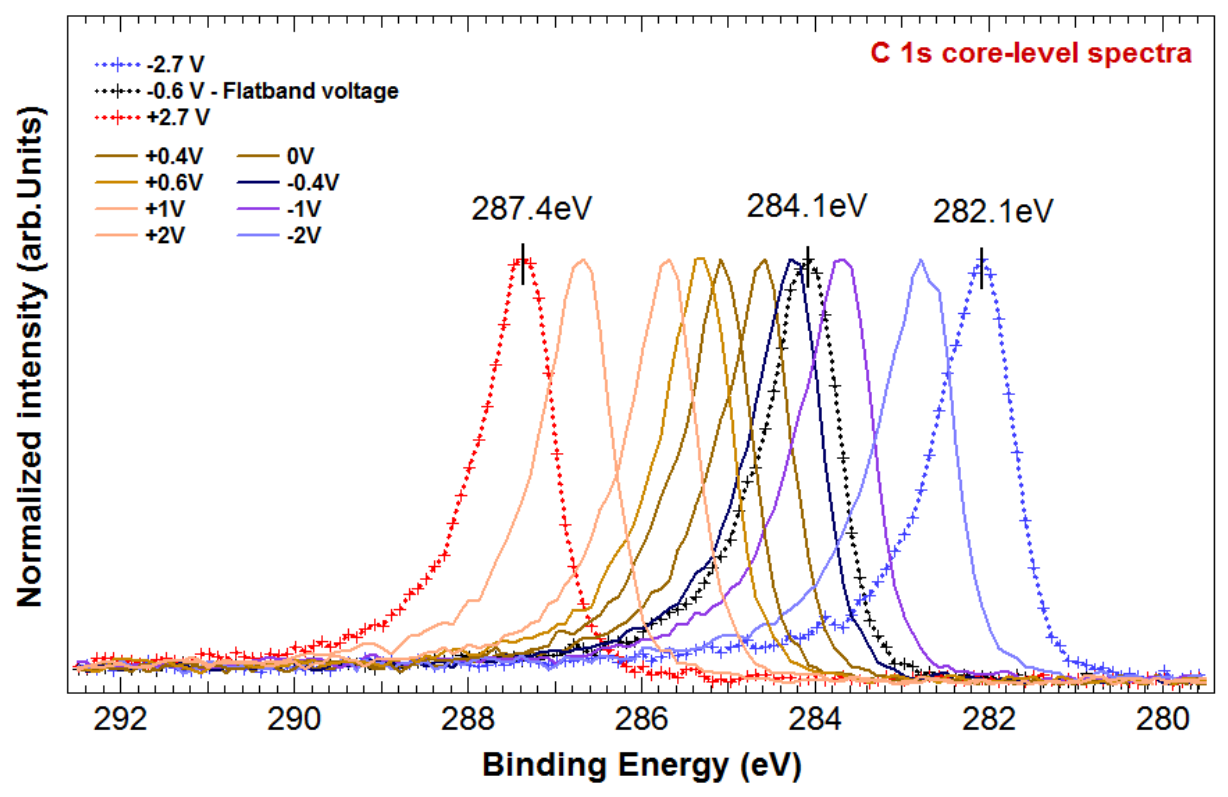

Figure S1: Bias-dependent C 1s core levels XPS spectra. 


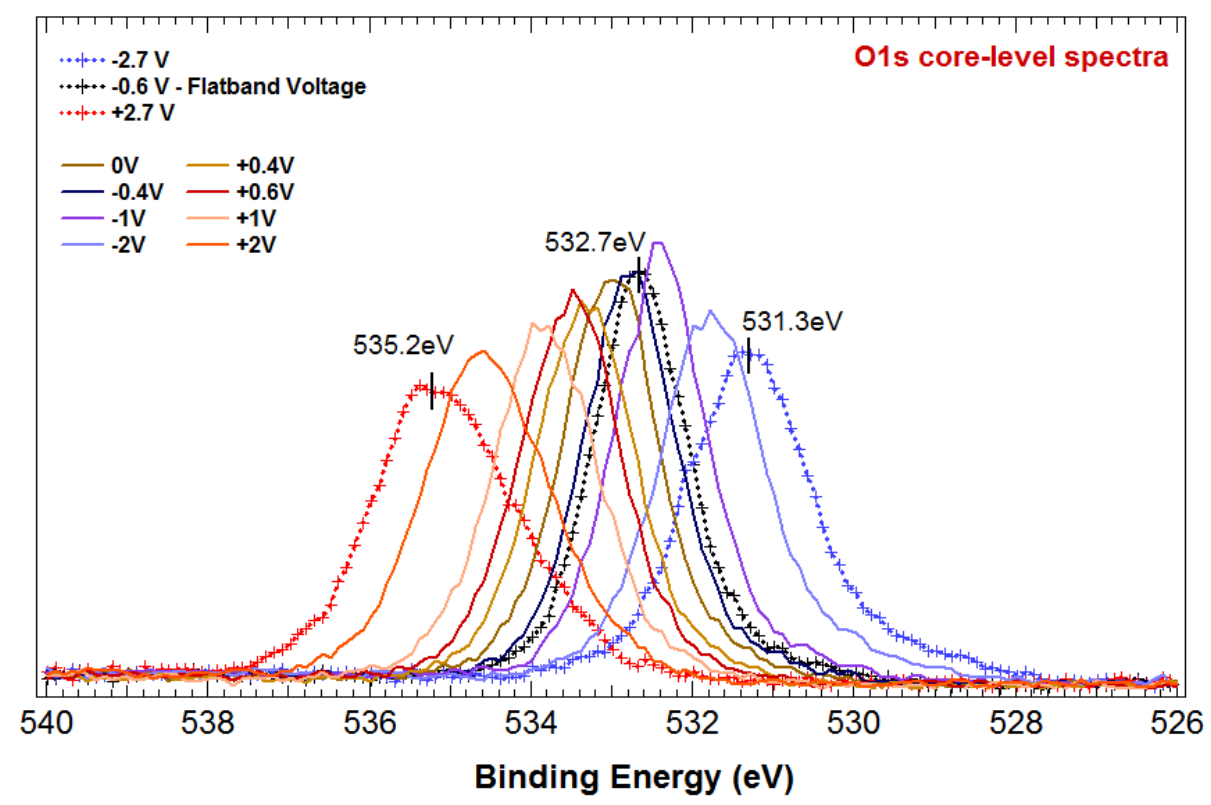

Figure S2: Bias-dependent O 1s core levels XPS spectra.

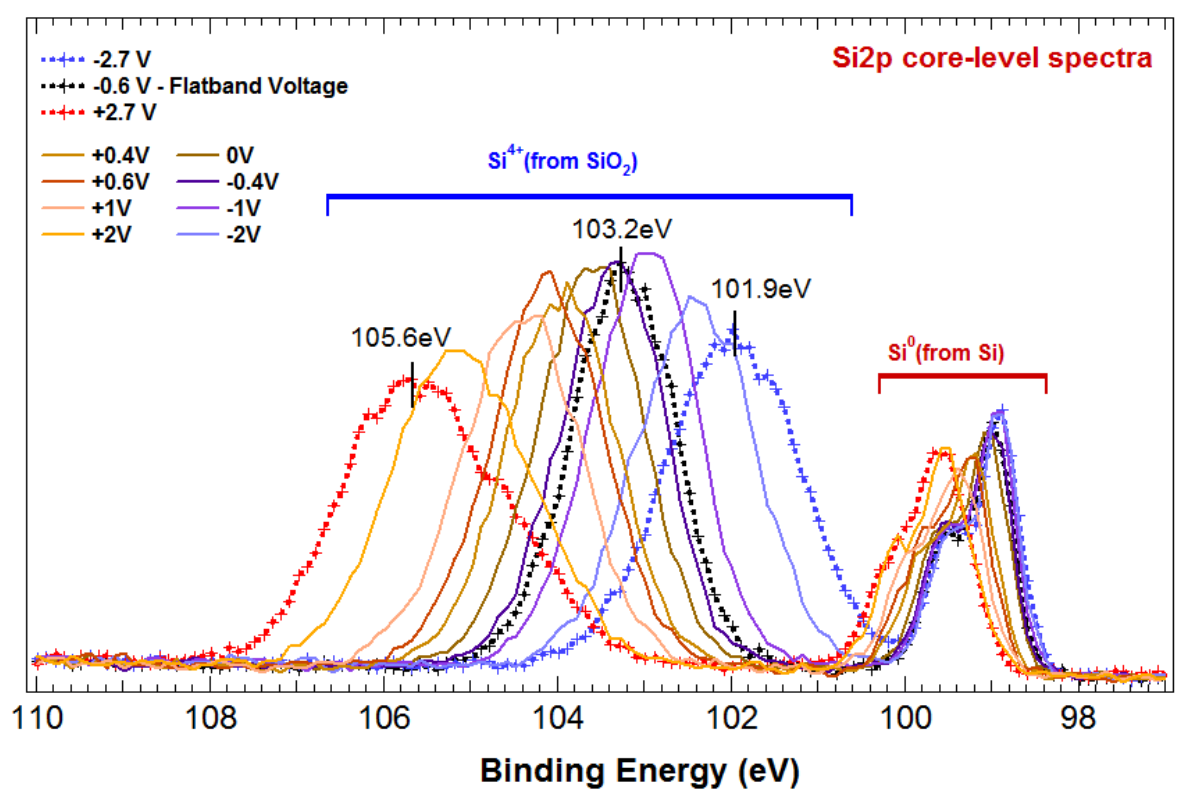

Figure S3: Bias-dependent Si 2p core levels XPS spectra.

\section{Energy alignment under biasing conditions}

It is possible to reconstruct, from the XPS information, the energy diagram across the $\mathrm{Gr} / \mathrm{SiO} 2 / \mathrm{Si}$ stack upon biasing. For this, we can use well-established values for the electron affinities, energy gaps and $\mathrm{Si}_{2} \mathrm{p}_{3 / 2}$ to valence band edge distances, for Si bulk and for $\mathrm{SiO}_{2} \cdot{ }^{7}$ 
Then the relative energy diagrams for the oxide and for $\mathrm{Si}$ are aligned using the position of the $\mathrm{Si} 2 \mathrm{p}_{3 / 2}$ core levels measured in this work $\left(\mathrm{Si}^{0}-\mathrm{Si}^{4+}\right.$ distance of $\left.4.3 \mathrm{eV}\right)$. The graphene side of the energy diagram is aligned using its Fermi level position.

In this work, we are using for Si: $\mathrm{EA}=4.1 \mathrm{eV}$, Egap $=1.1 \mathrm{eV}$ and $\mathrm{Si} 2 \mathrm{p}_{3 / 2}-\mathrm{VB}$ edge distance $=98.7 \mathrm{eV}$, and for $\mathrm{SiO}_{2}: \mathrm{EA}=1.3 \mathrm{eV}$, Egap $=8.9 \mathrm{eV}$ and $\mathrm{Si}_{2} \mathrm{p}_{3 / 2}-\mathrm{VB}$ edge distance $=98.6 \mathrm{eV}$. This leads to valence and conduction band offsets at the $\mathrm{SiO}_{2} / \mathrm{Si}$ interface of $4.4 \mathrm{eV}$ and 3.4 eV, respectively. Figures S4 and S5 compare the energy diagram obtained for flat band conditions and for accumulation and depletion conditions, respectively. Interface dipoles are usually calculated as the energy difference between bulk vacuum levels at each interface given an energy alignment configuration. Therefore at flat band, the interface dipole at the $\mathrm{SiO}_{2} / \mathrm{Si}$ interface is OS_ID $=0.6 \mathrm{eV}$, and the interface dipole at the $\mathrm{Gr} / \mathrm{SiO}_{2}$ interface is also GrO_ID $=0.6 \mathrm{eV}$ as shown on the left hand side of Figures S4 and S5.

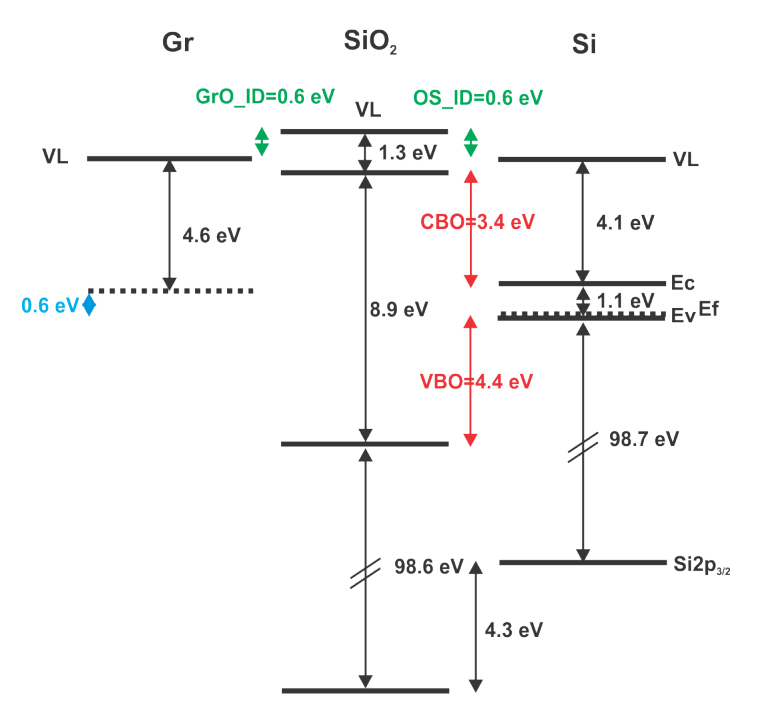

Flat band conditions

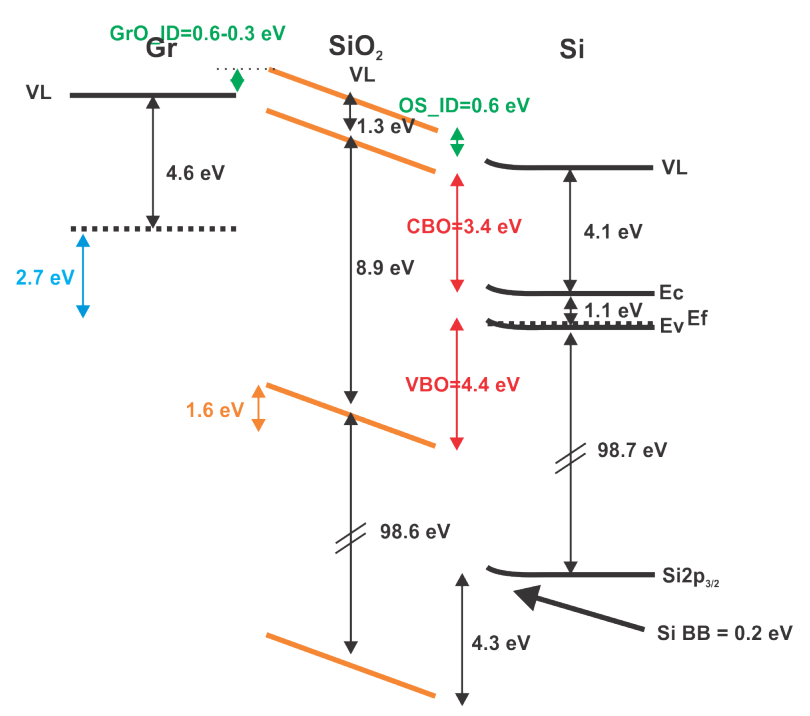

With Gr-SiO ${ }_{2}$ interfacial offset With band bending

Figure S4: Comparison between energy band diagram at flat band conditions ( $\mathrm{Vg}=-0.6 \mathrm{~V}$ ) and in accumulation condition $(\mathrm{Vg}=-2.7 \mathrm{~V})$.

When a gate voltage $\mathrm{Vg}=-2.7 \mathrm{~V}$ is applied, small band bending $(0.2 \mathrm{eV})$ is developing in $\mathrm{Si}$ and a small dipole $(0.3 \mathrm{eV})$ is created at the $\mathrm{Gr} / \mathrm{SiO}_{2}$ interface, reducing the total bias applied across the $\mathrm{SiO}_{2}$ by an amount of $0.5 \mathrm{eV}$ as depicted in the right hand side of Figure 
S4.

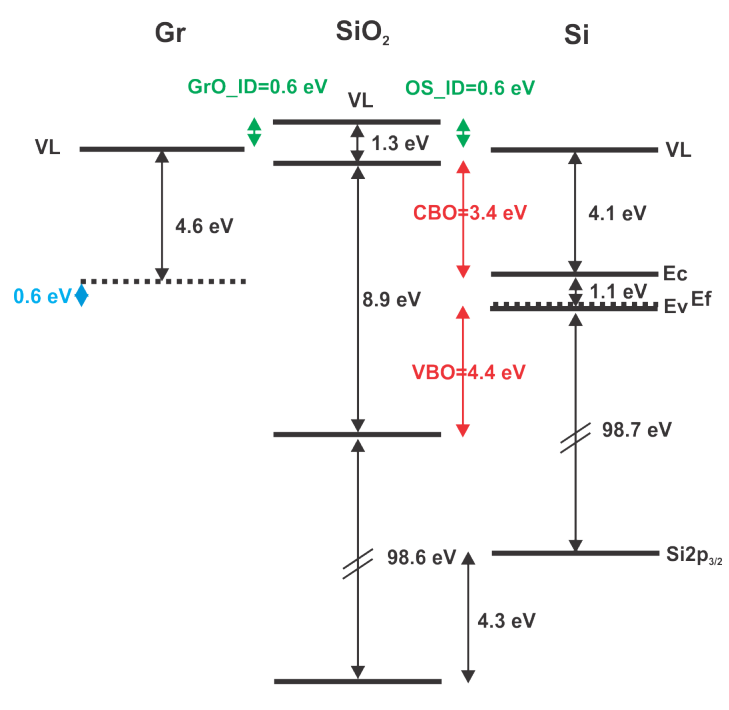

Flat band conditions

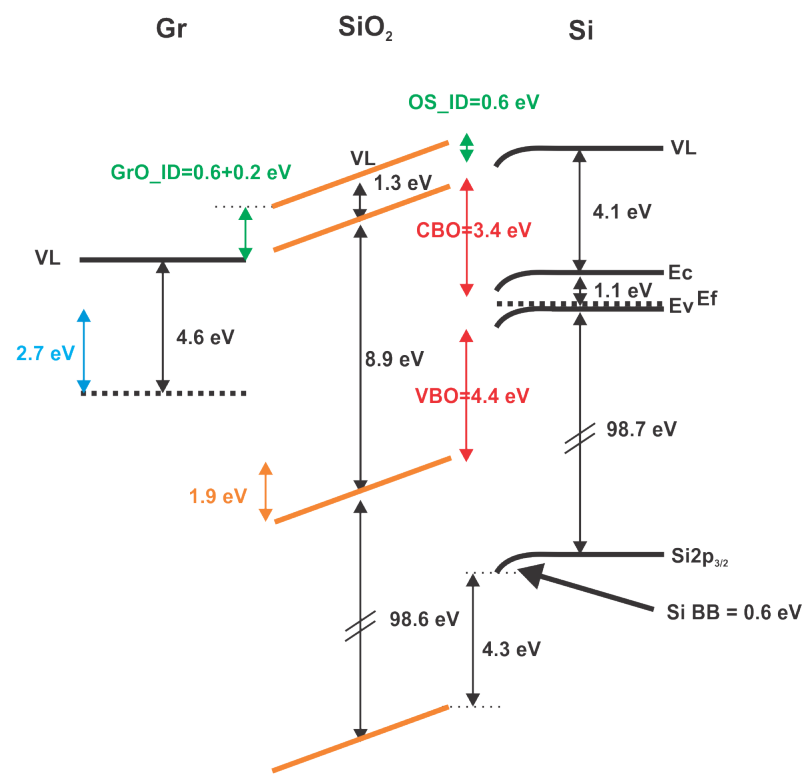

With $\mathrm{Gr}_{-} \mathrm{SiO}_{2}$ interfacial offset With band bending

Figure S5: Comparison between energy band diagram at flat band conditions $(\mathrm{Vg}=-0.6 \mathrm{~V})$ and in depletion condition $(\mathrm{Vg}=+2.7 \mathrm{~V})$.

When a gate voltage $\mathrm{Vg}=+2.7 \mathrm{~V}$ is applied, larger band bending $(0.6 \mathrm{eV})$ is developing in $\mathrm{Si}$ and small dipole $(0.2 \mathrm{eV})$ is created at the $\mathrm{Gr} / \mathrm{SiO}_{2}$ interface, reducing the total bias applied across the $\mathrm{SiO}_{2}$ by an amount of $0.8 \mathrm{eV}$ as depicted in the right hand side of Figure S5.

Note that in all cases, the valence and conduction band offsets at the $\mathrm{SiO}_{2} / \mathrm{Si}$ interface is an invariant. However, the interface dipole at the $\mathrm{Gr} / \mathrm{SiO}_{2}$ interface is always altered by the applied bias. Slides decomposing the transition from flat band to bias conditions are added to this SI file.

\section{References}

(1) Kern, W. Handbook of semiconductor wafer cleaning technology. New Jersey: Noyes Publication 1993, 111-196. 
(2) Luo, Z.; Kim, S.; Kawamoto, N.; Rappe, A. M.; Johnson, A. C. Growth mechanism of hexagonal-shape graphene flakes with zigzag edges. ACS Nano 2011, 5, 9154-9160.

(3) Wu, W.; Jauregui, L. A.; Su, Z.; Liu, Z.; Bao, J.; Chen, Y. P.; Yu, Q. Growth of single crystal graphene arrays by locally controlling nucleation on polycrystalline $\mathrm{Cu}$ using chemical vapor deposition. Adv. Mater. 2011, 23, 4898-4903.

(4) Ballan, H.; Declercq, M. High voltage devices and circuits in standard CMOS technologies; Springer Science \& Business Media, 2013.

(5) Powell, C. J.; Jablonski, A. NIST Electron Effective-Attenuation-Length Database, Version 1.3, SRD 82, National Institute of Standards and Technology, Gaithersburg, MD. 2011

(6) Vitchev, R.; Defranoux, C.; Wolstenholme, J.; Conard, T.; Bender, H.; Pireaux, J.-J.

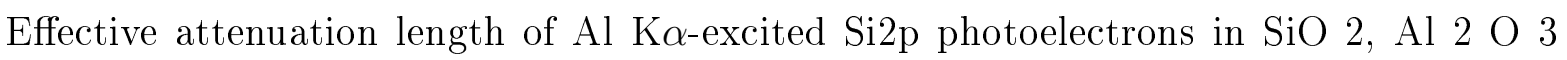
and HfO 2 thin films. J. Electron Spectrosc. 2005, 149, 37-44.

(7) Bersch, E.; Rangan, S.; Bartynski, R. A.; Garfunkel, E.; Vescovo, E. Band offsets of ultrathin high- $\kappa$ oxide films with Si. Phys. Rev. B 2008, 78, 085114. 